\title{
Evaluation of an Image-based Automated Detection System in Detecting Ki67 Proliferation Index and Correlation with the Traditional Eye-Ball Method in Gastroenteropancreatic Neuroendocrine Tumors
}

\author{
Yelda Dere1, Osman Ozkaraca², Gurcan Cetin² and Ozcan Dere³
}

\begin{abstract}
Objective: To design an application which can calculate Ki67 and compare its results with the traditional method in gastroenteropancreatic (GEP)-neuroendocrine tumors (NETs).

Study Design: Descriptive analytical study.

Place and Duration of Study: Faculties of Medicine and Technology of Mugla Sitki Kocman University between January 2015 to January 2016.

Methodology: A new analyser for detecting the exact percentage of positive cells in images captured from different slides retrospectively selected from hospital records was designed and the concordance with results given by an expert pathologist was compared. Demonstrative slides from randomly selected 50 patients diagnosed as GEP-NETs were stained with Ki67 antibody; and images were captured from the hotspots. The images were then uploaded to the application of the analyser designed for detecting the percentage of Ki67-stained cells.

Results: Twenty-seven male (54\%) and $23(46 \%)$ female patients with a mean age of $52.3 \pm 8.80$ years were included. According to the pathologist with eye-ball method, 17 cases were grade $1(34 \%), 21$ cases were grade $2(42 \%)$ and $12(24 \%)$ cases were grade 3. By software, 8 cases were grade $1(16 \%), 36$ cases were grade $2(72 \%)$ and 6 cases were grade $3(12 \%)$. Statistical evaluation revealed a kappa value of 0.447 indicating moderate aggreement between the pathologist and the software.

Conclusion: The total count of the cells both by the analyser and the pathologist were similar. However, improvements are needed to raise the precision for the detection of positive and negative tumoral cells.
\end{abstract}

Key Words: Neuroendocrine tumor, Ki67, Neuroendocrine tumor grade, Automated detection system.

\section{INTRODUCTION}

Gastroenteropancreatic neuroendocrine tumors (GEPNETs) are neuroendocrine tumors characterised with uniform cells and bland cytological features. With the latest updates of WHO Classification of Tumors, NETs are graded as G1, G2 and G3 according to the proliferative index of the tumor evaluated by Ki67 antibody. ${ }^{1}$

Grading of the neuroendocrine tumors depends on their mitotic activity and Ki67 proliferation index. ${ }^{1} \mathrm{Ki} 67$ proliferation index was strictly graded as $<3 \%$ grade 1 , $3-20 \%$ grade $2,>20 \%$ grade 3.1 But the detection of these grades may cause problems, especially in borderline cases. Many detection methods were offered by different authors. The most commonly used technique for determining Ki67 proliferation index is the hotspot counting depending on the advantages of being the cheapest and the fastest method. However, the reproducibility and the interobserver agreement rates can be low.

Department of Pathologyl / Information Systems Engineering2/ Surgery ${ }^{3}$, School of Medicine, Mugla Sitki Koçman University, Mugla, Turkey

Correspondence: Dr. Yelda Dere, Department of Pathology,

School of Medicine, Mugla Sitki Koçman University, Mugla, Turkey

E-mail:yeldamorgul@gmail.com

Received: March 20, 2018; Accepted: July 28, 2018
Image analysers are another option for counting Ki67 index.2-4 The use of these methods also changes depending on the technological facilities of the pathology laboratories worldwide; and due to expensive charges, it does not seem possible to set these devices in every laboratory, especially in countries with economical issues.

Depending on all these disadvantages and advantages of these methods, none of them was accepted globally as gold standard. In the study of Reid et al., eye-count on captured/printed images were found as the most cost-effective and reliable method. 5

Desktop computers or laptops are one of the musts in every pathology laboratory and for every pathologist even in countries with low socioeconomic level. Designing a simple but effective software suitable for every computer would help pathologists define accurate results in many different tumors, grading of tumors or any type of cell calculation.

The aim of this study was to design a simple software which can load captured images right from the microscopic camera application with no additional device, count the immunostained cells and calculate the percentage in addition to compare its results with eyeball estimation on these captured images. 


\section{METHODOLOGY}

Ethical approval was taken from Mugla Sitki Koçman University Ethical Committee for Clinical Trials and this study was supported by a project from the Scientific Research Projects Management Unit of Mugla Sitki Koçman University (Grant number: 15/097).

This descriptive study was conducted at Faculties of Medicine and Technology of Mugla Sitki Kocman University between January 2015 to January 2016. Randomly selected 50 GEP-NETs from the archives of the pathology laboratory of the study centre were included in this study. All of these tumors were diagnosed and graded by hotspot counting method. The size of the tumors and the types of the excision procedures were obtained from the pathology reports. For Ki67 immunostaining, 3-4 mm thick sections were cut from the paraffine blocks and the immunostaining procedure was held automatically by Leica Bond-Max using anti-Ki67 antibody (Leica).

Ki67 stained slides were revised, and on x20 objective, the highest nuclear labelled area "hotspot" was selected and microphotograph was captured by using Leica MC120 HD camera attached to DM1000 LED microscope. Images were then loaded to the software designed by Technology Faculty of our instution for Ki67 positive cell counting.
All images were revised by eye-counting percentage of positively stained cells in the hotspot by the same pathologist and the results given by the software and the pathologist was compared for variability.

An algorithm for disassemble positive and negative cells using K-means clusters was developed in this software for detecting Ki67 proliferation index. The images and cell borders were relatively not easily defined in some areas. In such images, Hough transformation was added for increasing the cell detection success. This software, which can be run on a standard office desktop computer/ laptop with a processor of Intel i5 $3.20 \mathrm{Ghz}$ and $8 \mathrm{~GB}$ video card, can analyse $1360 * 1024$ pixel and .jpeg formatted microscopic images in about 2 seconds and calculate the percentage of cells which gives Ki67 proliferation index.

Two different observers (the pathologist and the software) gave the percentage of Ki67 positive cells and these results were regrouped according to WHO GEPNET grading scale as $<3 \%=$ Grade $1,3-20 \%=$ Grade 2 and $>20 \%=$ Grade 3 . Descriptive statistics are reported as the mean $\pm S D$ and categorical variables were given as frequencies (percentages). A p-value less than and equal to $0.05(p \leq 0.05)$ was considered as statistically significant. Frequencies and descriptive statistics were run by SPSS $\vee 20.0$ and kappa statistics were run by $R \vee 3.2 .4$ and Cohen's kappa was calculated for interobserver variability.

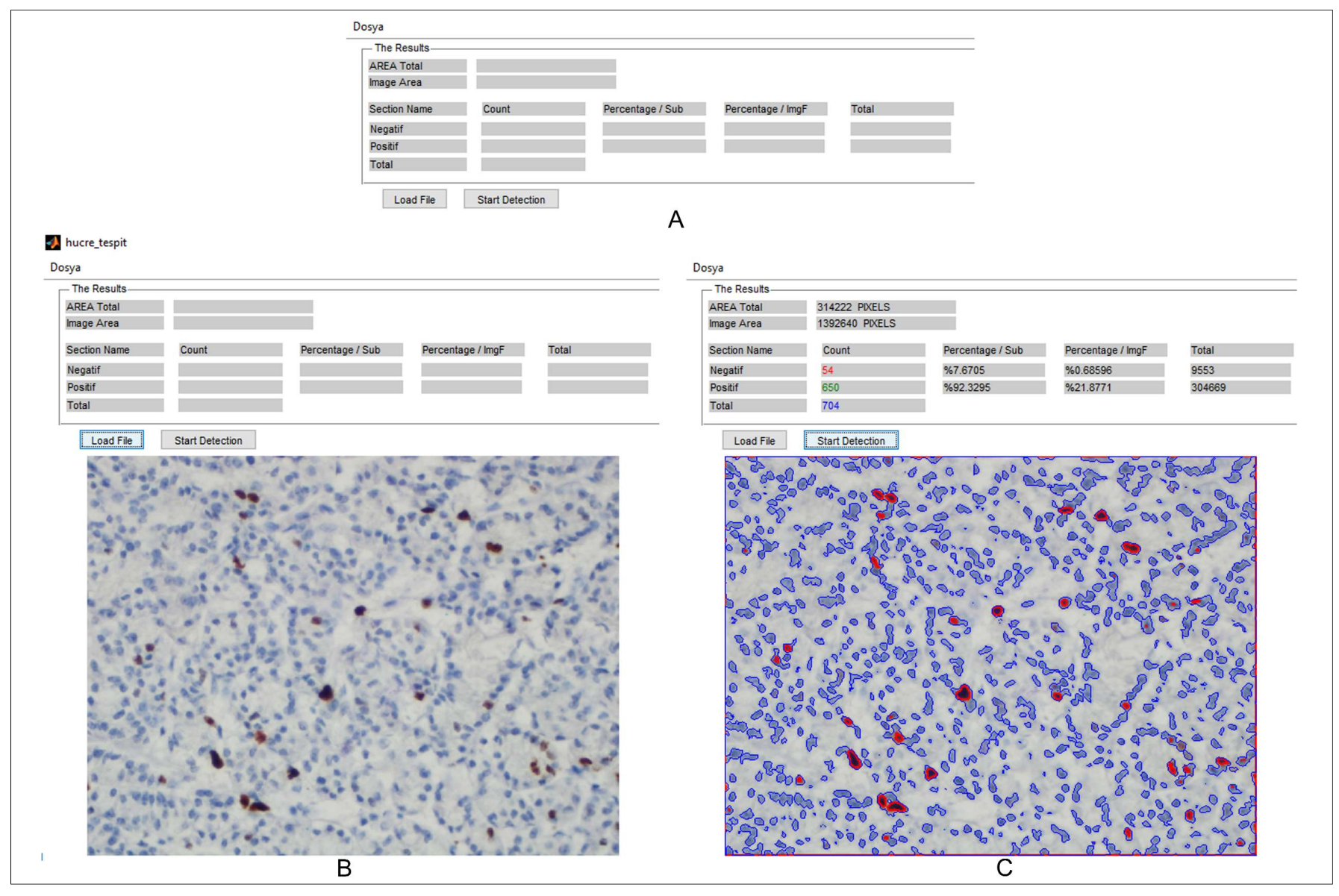

Figure 1: The screen views of the analyser: (A) Start-up screen; (B) An image loaded in the application (C) result screen. 


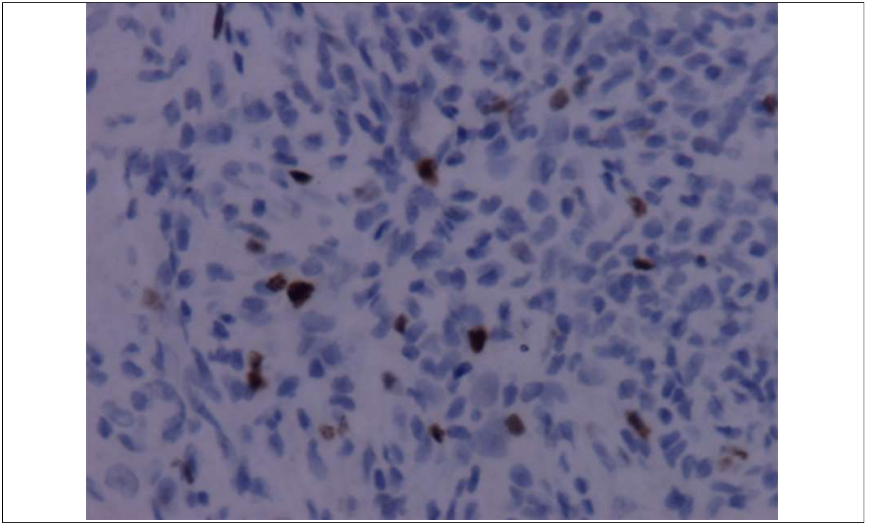

Figure 2: Neuroendocrine tumor, Grade 1, DAB, X40

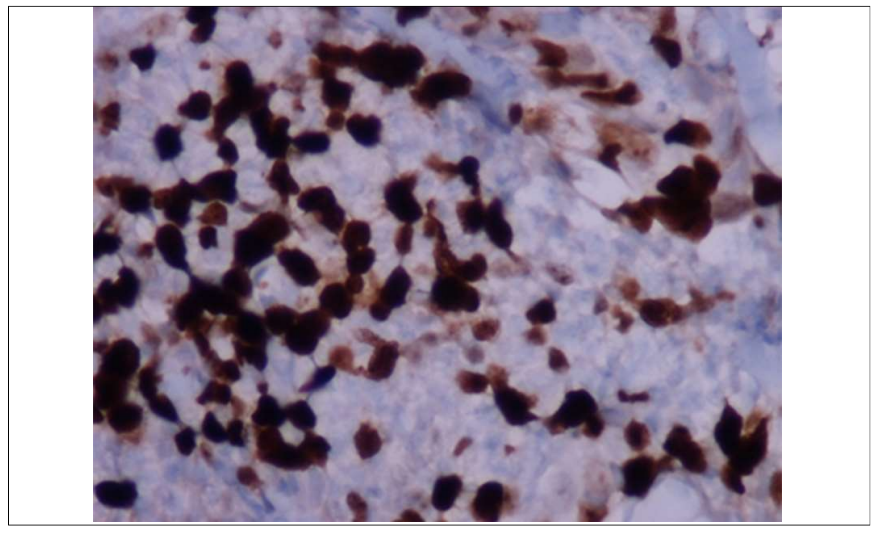

Figure 3: Neuroendocrine tumor, Grade 3, DAB, X40.

\section{RESULTS}

Among 50 GEP-NETs, 27 were males (54\%) while 23 were females $(46 \%)$. The mean age of the patients was $52.3 \pm 8.80$ years. Patients were diagnosed by endoscopic biopsy in 38 cases (76\%); and 12 cases $(24 \%)$ were diagnosed by surgical excision. The average size of the surgically excised tumors was $2.8 \pm 0.66 \mathrm{~cm}$. Among all tumors, $26(52 \%)$ were located in the stomach, $10(20 \%)$ were appendiceal, $3(6 \%)$ were colorectal, 3 $(6 \%)$ were ileal, $8(16 \%)$ were pancreatic NETs.

The images were analysed for both the total count of all cells, immunohistochemically stained cells and the percentage of these cells. After counting, the pathologist calculated the percentage. However, the analyser programme gave the percentage automatically. The main screen views of the analyser was given in Figure 1.

Agreement statistic of Cohen's kappa was used while comparing the percentages of the stained and unstained cells so as to grade the tumor. According to Ki67 index calculated by the pathologist with hotspot eye-counting method; 17 cases were grade $1(34 \%), 21$ cases were grade $2(42 \%)$, and 12 cases were grade $3(24 \%)$. By software, 8 cases were grade $1(16 \%), 36$ cases were Grade $2(72 \%)$ and 6 cases were grade 3 $(12 \%)$. Statistical evaluation revealed a kappa value of 0.447 indicating moderate aggreement between the pathologist and the software.
Table I: The distribution of grades given by the pathologist and the analyser.

\begin{tabular}{l|ccc|c}
\hline \multirow{2}{*}{ Analyser's grade } & \multicolumn{3}{|c|}{ Pathologist's grade } & \multirow{2}{*}{ Total } \\
\cline { 2 - 4 } & 1 & 2 & 3 & \\
\hline 1 & $8(16 \%)$ & 0 & 0 & $8(16 \%)$ \\
2 & $9(18 \%)$ & $20(40 \%)$ & $7(14 \%)$ & $36(72 \%)$ \\
3 & 0 & $1(2 \%)$ & $5(10 \%)$ & $6(12 \%)$ \\
\hline Total & $17(34 \%)$ & $21(42 \%)$ & $12(24 \%)$ & $50(100 \%)$ \\
\hline
\end{tabular}

Among these 50 cases, $33(66 \%)$ were consensus cases in both the pathologist and the software found the same grade. Among consensus cases, 8 were grade 1 (Figure 2, 16\%), 20 were grade $2(40 \%)$ and 5 were grade 3 (Figure 3, 10\%, Table I).

\section{DISCUSSION}

Determining Ki67 index in NETs is a common problem in surgical pathology practice. Because of the strict cut-off values of WHO grading of NETs, it is even more important to give distinct results. ${ }^{1}$

Different methods were offered in the literature for evaluating accurate Ki67 index because of the low interobserver aggreement rates worldwide not just for GEP-NETs but also in various tumor types.6-8 Some of these methods were accompanied with automated image analysers. Automated analysers scan the whole slide and trained personnel (technicians or pathologists) choose the "hotspots" and the selected areas were then counted automatically. This method reduces time for evaluation, and is accepted as gold standard but the whole system is expensive to use in every laboratory in every country. 9,10

Automated counting by image analysers scan the whole slide, choose the hotspots and count the Ki67 index. In different studies Aperio immunohistochemical nuclear quantifier and Nuclear v9 image analysis algorithm were used for digital image analysis.11-13 But in these algorithms, cut off for the size and the shape of the tumor cells were controlled manually because of the need to exclude stromal cells and lymphocytes and this increases the duration of an analysis of an image up to 10 minutes. In automatic counting analysers such as ImageJ,14 where macro-based markers of different colors are being used, cannot give very accurate results due to fragmented cells.

But these methods need trained technicians who can choose hotspots and this automated analysers are generally used in reference laboratories of Turkey, which have more technical and financial support. In other centres, these analysers are not accepted because of the high cost. Many other methods are still being investigated for detecting exact percentage, which is one of the most important factors for neuroendocrine tumors. 6 Bologna-Molina et al. suggested a method using only a digital camera attached microscope and a computer. In this method, they offered to use a grid in table covering the immunohistochemical image and manually count the cells. ${ }^{15}$ However, these methods generally take time to get to the results. 
Eye-balling, fastest and the cheapest method, is the most commonly used method for counting Ki67.16 Although widely used and known as the most practical method, eye-balling has disadvantages such as high inter and intraobserver variability rates and low reproducibility in especially borderline cases. Even though some authors offered this method as reliable, unexperienced pathologists may not overcome the problem of this variability.

In the study by Reid et al., manual Ki67 counting upon camera captured/printed images was offered. The slides of tumors were revised and images from the hotspots were then captured by an attached camera. Ki67 positive and negative cells were counted from the printed images and the percentage was calculated. 5 In this study, it was also captured images from hotspots but transfer the images to the software loaded at the same computer and the software counted and calculated the percentage of Ki67 positive cells. The target of this software was to reduce time loss while counting manually. However a kappa value of 0.447 is not acceptable in this type of study but similar with other studies found in the literature such as Dhall et al. and Tang et al. reported.9,16 In the study of Tang et al., a kappa value of 0.24 (moderate aggreement) was calculated between the analyser and the observer similar to the kappa value of 0.39 which was reported in the study of Dhall et al.9,16 Moderate agreement may be due to the overlapping of the cells in the images on which eye-balling can differ but the software cannot. In addition to that, the heterogenity of the tumors can also affect the results. For example, high tumoral cellularity may mask the real positive cells that can result undergrading. ${ }^{17,18}$ This phenomenon can also be observed in other types of tumors such as breast cancer samples. ${ }^{19,20}$

\section{CONCLUSION}

Different methods or analysers can be used in detecting positively stained cells, but there should be higher agreement rates when compared with experts.

\section{REFERENCES}

1. Rindi G, Arnold R, Bosman FT. Nomenclature and classification of neuroendocrine neoplasms of the digestive system in WHO Classification of Tumors of the Digestive System, $4^{\text {th }}$ ed, 2010; p.13-4.

2. Markiewicz T. Using MATLAB software with Tomcat server and Java platform for remote image analysis in pathology. Diagn Pathol 2011; 6(Suppl):S18.

3. Grala B, Markiewicz T, Kozlowski W, Osowski S, Slodkowska J, Papierz W. New automated image analysis method for the assessment of Ki-67 labeling index in meningiomas. Folia Histochem Cytobiol 2009; 47:587-92.

4. Xing F, Su H, Neltner J, Yang L. Automatic Ki-67 counting using robust cell detection and online dictionary learning. IEEE Trans Biomed Eng 2014; 61:859-70.
5. Reid MD, Bagci P, Ohike N, Saka B, Seven IE, Dursun N. Calculation of the Ki67 index in pancreatic neuroendocrine tumors: A comparative analysis of four counting methodologies. Mod Pathol 2015; 28:686-94.

6. Laurinavicius A, Plancoulaine B, Laurinaviciene A, Herlin P, Meskauskas R, Baltrusaityte I, et al. A methodology to ensure and improve accuracy of Ki67 labelling index estimation by automated digital image analysis in breast cancer tissue. Breast Can Res 2014; 16:R35.

7. Knutsvik G, Stefansson IM, Aziz S, Arnes J, Eide J, Collett K. Evaluation of Ki67 expression across distinct categories of breast cancer specimens: A population-based study of matched surgical specimens, core needle biopsies and tissue microarrays. PLoS One 2014; 9:e112121.

8. Warth A, Fink L, Eckhoff AF, Jonigk D, Keller M, Ott G. Interobserver agreement of proliferation index (Ki-67) out-performs mitotic count in pulmonary carcinoids. Virchows Arch 2013; 462:507-13

9. Dhall D, Frishberg DP, Galliano G. Interobserver variability in assessing ki-67 proliferative index in gastrointestinal welldifferentiated neuroendocrine neoplasms. Mod Pathol 2009; 22:116.

10. Goodell PP, Krasinskas AM, Davison JM, Hartman DJ. Comparison of methods for proliferative index analysis for grading pancreatic well-differentiated neuroendocrine tumors. Am J Clin Pathol 2012; 137:576-82.

11. Adams RW, Dyson P, Barthelmes L. Neuroendocrine breast tumours: Breast cancer or neuroendocrine cancer presenting in the breast? Breast 2014; 23:120-7.

12. Dhall $D$, Mertens R, Bresee C. Ki-67 proliferative index predicts progression-free survival of patients with well-differentiated ileal neuroendocrine tumors. Hum Pathol 2012; 43:489-95.

13. Romero Q, Bendahl P-O, Fernö M. A novel model for Ki67 assessment in breast cancer. Diag Pathol 2014; 9:118.

14. Giesel FL, Kratochwil C, Mehndiratta A. Comparison of neuroendocrine tumor detection and characterization using DOTATOC-PET in correlation with contrast enhanced CT and delayed contrast enhanced MRI. Eur J Radiol 2012; 81: 2820-5.

15. Bologna-Molina R, Damián-Matsumura P, Molina-Frechero N. An easy cell counting method for immunohistochemistry that does not use an image analysis program. Histopathol 2011; 59:801-3.

16. Tang LH, Gonen M, Hedvat C. Objective quantification of the Ki67 proliferative index in neuroendocrine tumors of the gastroenteropancreatic system: A comparison of digital image analysis with manual methods. Am J Surg Pathol 2012; 36: 1761-70.

17. Modlin IM, Oberg K, Chung DC. Gastroenteropancreatic neuroendocrine tumours. Lancet Oncol 2008; 9:61-72

18. Tang LH, Klimstra DS. Conundrums and caveats in neuroendocrine tumors of the pancreas. Surg Pathol Clin 2011; 4: 589-625.

19. Friedberg RC, Pantanowitz L. Practice evolution: Decentralized computer-assisted immunohistochemical image analysis. Arch Pathol Lab Med 2009; 133:597-600.

20. Fasanella S, Leonardi E, Cantaloni C, Eccher C, Bazzanella I, Aldovini $D$, et al. Proliferative activity in human breast cancer: $\mathrm{Ki}-67$ automated evaluation and the influence of different Ki-67 equivalent antibodies. Diagn Pathol 2011; 6:1-7.

\section{$\cdots \cdot \cdot \hat{\tau} \cdot \cdots$}

